\title{
Expression of tissue inhibitors of metalloproteinase 1 (TIMP-1) in gastric cancer tissue
}

\author{
Barbara Mroczko', Marta Lukaszewicz-Zając ${ }^{1}$, Magdalena Groblewska1, \\ Jolanta Czyżewska', Mariusz Gryko ${ }^{3}$, Katarzyna Guzińska-Ustymowicz, \\ Andrzej Kemona ${ }^{4}$, Bogusław Kędra ${ }^{3}$ and Maciej Szmitkowski ${ }^{1}$ \\ ${ }^{1}$ Department of Biochemical Diagnostics, ${ }^{2}$ Department of Clinical Laboratory Diagnostics, \\ ${ }^{3}$ Second Department of General Surgery, ${ }^{4}$ Department of General Pathomorphology, \\ Medical University, Białystok, Poland.
}

\begin{abstract}
Degradation of extracellular matrix (ECM) is an essential step of invasion and metastasis of gastric cancer. The proteolysis of basement membranes depends on the balance between activities of matrix metalloproteinases (MMPs) and their tissue inhibitors (TIMPs). The aim of the study was to assess the expression of TIMP-1 in gastric cancer (GC) and interstitial inflammatory infiltrate cells within GC tissue in relation to clinico-pathological features of tumor and to estimate the prognostic significance of TIMP-1 expression for patients' survival. The presence of TIMP-1 in 54 cases of gastric cancer samples was investigated by immunohistochemistry. The expression of TIMP-1 in cancer and interstitial inflammatory infiltrate cells was evaluated in semi-quantitative scale. The immunoreactivity of TIMP-1 in cancer and inflammatory cells was positive in $100 \%$ of cases and varied from weak to intense reaction. The intensity of TIMP-1 expression increased with more advanced tumor stages and in patients who died of cancer during 2-year observation. TIMP-1 expression in interstitial inflammatory infiltrate cells was the independent prognostic factor for patients' survival. The results suggest the role of TIMP-1 in gastric tumorigenesis, although this issue requires further investigtions.
\end{abstract}

Key words: gastric cancer, tissue inhibitor of matrix metalloproteinase 1

\section{Introduction}

Gastric cancer (GC) is one of the malignant tumors of gastrointestinal tract with the highest mortality [1]. Degradation of extracellular matrix (ECM), the physical barrier for migration of neoplastic cells into tissues, is known as an essential step of tumor invasion, aggressive growth and formation of metastases $[2,3]$. Matrix metalloproteinases (MMPs) are the enzymes capable of degrading all the components of ECM [4]. The proteolysis of basement membranes depends on the balance between activities of MMPs and tissue inhibitors of metalloproteinases (TIMPs) [3]. Moreover, TIMPs play an important role in the extent of the matrix degradation [5].

Enhanced tissue expression of TIMP-1 was observed in various malignant tumors, including

Correspondence: B. Mroczko, Dept. of Biochemical Diagnostics, Medical University, Białystok, Waszyngtona 15 a, 15-269 Białystok, Poland; tel.: (+4885) 7468710, fax.: (+4885) 7468585, e-mail: mroczko@umwb.edu.pl gastric [6] and colorectal cancers [7]. It has been also shown that expression of TIMP-1 in GC tissue correlated with tumor invasion and lymph node metastasis [8]. However, little is known about expression of TIMP-1 in interstitial inflammatory infiltrate cells in gastric cancer. Infiltration of inflammatory cells is a characteristic feature of many malignant tumors, including gastric cancer. The inflammatory cells produce metalloproteinases to the peritumoral environment. These cells are also able to synthesize various cytokines enhancing expression of MMPs and TIMPs by both - tumoral and stromal cells [9].

Therefore, the aim of the current study was to compare the intensity of TIMP-1 expression in neoplastic and interstitial inflammatory infiltrate cells in gastric cancer tissue with clinico-pathological features of tumors. According to our knowledge, this is the first study assessing the expression of TIMP-1 in tumor-infiltrating lymphocytes and other inflammatory cells within GC tissue in relation to patients' survival. 


\section{Material and methods}

Patients. Fifty-four GC patients diagnosed from April 2003 to May 2008 and operated on in the Second General Surgery Departament, University Hospital of Białystok were studied. The primary selection criteria for this study were the adequate follow-up for tumor-specific analysis of survival and the availability of tumor samples (formalin-fixed and parafin-embedded blocks).

The study group included 13 women and 41 men, mean age was 67.5 year (range 37-84 years). Clinical diagnosis of gastric cancer was confirmed by microscopic examination of the material obtained during surgery or endoscopy. The standard TNM (tumor, nodulus, metastases) classification was used for staging of tumors according to the 5th International Union Against Cancer [10]. Thirty-five patients survived, whereas 19 patients died of cancer during the observation period (1-48 months). The study was approved by the local Ethics Committee. All the patients gave their informed consent for the analysis.

Immunohistochemical (IHC) staining. The tissue samples were collected during the course of surgery, fixed in $10 \%$ buffered formalin, dehydrated and embedded in paraffin. For detection of TIMP-1 expression in cancer tissue the mice monoclonal antibodies against human TIMP-1 (Novocastra; clone 6F6a, dilution 1:112) were used. The visualization of antigen presence was obtained using Novocastra Peroxidase Detection System. The expression of TIMP-1 in gastric cancer cells as well as in inflammatory infiltrate cells (macrophages, polinuclears and lymphocytes) in the neoplastic interstitium was evaluated in the light microscopy, using semi-quantitative scale: $0 \mathrm{pt}-$ no reaction, $1 \mathrm{pt}$ - weak, 2 pts - moderate and 3 pts - intense reaction.

Statistical analysis. Data are presented as number of cases and percent of analyzed subgroup. Comparisons between subgroups were made using Fisher-Freeman-Halton's test [11]. Univariate analyses of survival were performed using the log-rank test, and multivariate analyses employed the Cox proportional hazards model. The differences were considered as statistically significant with $p<0.05$. Statistical analyses were carried out using the STATISTICA 5.1 PL program (StatSoft Inc., Tulsa, OK).

\section{Results}

\section{The expression of tissue inhibitor of matrix metalloproteinases-1 in gastric cancer cells}

The positive reaction for TIMP-1 was observed in $100 \%$ of all tissue samples of gastric cancer: weak (1 pt) in $22 \%$, moderate ( 2 pts) in $50 \%$ and an intense expression ( 3 pts) in $28 \%$ of cases (Fig. 1). The results of immunostaining for the presence of TIMP-1 in neoplastic cells in relation to clinico-pathological features of tumor are presented in Table 1. The number of moderate $(2 \mathrm{pts})$ and intense reactions ( $3 \mathrm{pts})$ increased with tumor stage and was the highest in stage IV $(56 \%$ and $44 \%$, respectively).

Similar results were observed for relationships between the intensity of TIMP-1 expression and the depth of tumor invasion ( $\mathrm{T}$ factor). Number of cases with intense expression of this protein was higher in T4 subgroup (50\%) than in T2 and T3 samples (18\% and $23 \%$, respectively). Moreover, there were no weak reactions in T4 subgroups $(0 \%)$. The percentages of intense expression of TIMP-1 in cancer cells increased with the

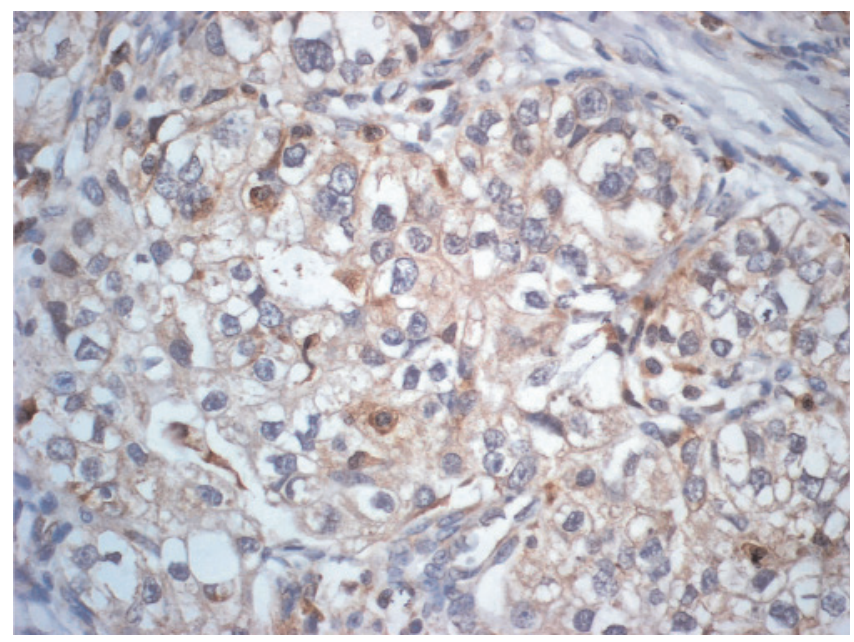

Fig. 1. TIMP-1 moderate expression in gastric cancer cells (2 pts).

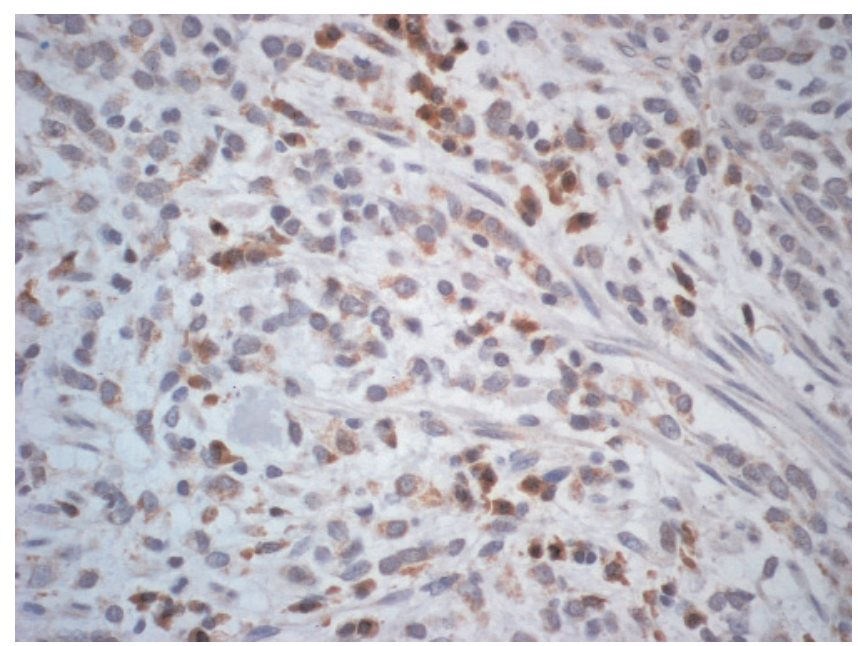

Fig. 2. TIMP-1 moderate expression in inflammatory infiltrate cells (2 pts).

number of metastatic lymph nodes. It was observed in $6 \%$ of patients from N0 subgroup, $22 \%$ in N1, 31\% of N2 and $54 \%$ of patients from N3 subgroup. The highest percent of weak reaction (1 pt) was observed in N0 subgroup (44\%). It was shown that expression of TIMP-1 in cancer cells depended on the presence of distant metastases. There were almost two times more cases with intense reactions $(42 \%)$ in the M1 subgroup of GC samples than in the M0 samples (24\%). Moreover, there were no cases ( $0 \%)$ with weak intensity of immunostaining in M1 subgroup in comparison with $29 \%$ in M0.

The intensity of TIMP-1 expression in cancer cells correlated with survival of patients. Intense (3 pts) expression in tumor samples from patients who died of cancer during follow-up period was seven times higher $(63 \%)$ than in samples from patient who survived $(9 \%)$.

The mean value of TIMP-1 expressions in gastric cancer cells were higher in more advanced stages of 
Table 1. The expression of TIMP-1 in gastric cancer cells in relation to clinico-pathological features of tumor.

\begin{tabular}{|c|c|c|c|c|c|c|}
\hline \multirow{2}{*}{\multicolumn{2}{|c|}{ Group tested }} & \multicolumn{3}{|c|}{ Cases $(\%)$} & \multirow{3}{*}{$\begin{array}{c}\text { Mcan } \\
\text { value }\end{array}$} & \multirow{3}{*}{$\mathrm{p}$} \\
\hline & & \multirow{2}{*}{$\frac{+}{7(50 \%)}$} & \multirow{2}{*}{$\frac{++}{5(38 \%)}$} & \multirow{2}{*}{$\frac{+++}{2(14 \%)}$} & & \\
\hline \multirow{3}{*}{$\begin{array}{l}\text { Tumor stage } \\
\text { (INM classification) }\end{array}$} & $\mathrm{I}+\mathrm{II}(\mathrm{n}=14)$ & & & & & \\
\hline & III $(n=22)$ & $5(23 \%)$ & $12(55 \%)$ & $5(23 \%)$ & 2.00 & \multirow[t]{2}{*}{$0.011 *$} \\
\hline & $\operatorname{IV}(n=18)$ & $0(0 \%)$ & $10(56 \%)$ & $8(44 \%)$ & 2.44 & \\
\hline \multirow{3}{*}{ Depth of tumor invasion } & $12(n=11)$ & $6(55 \%)$ & $3(27 \%)$ & $2(18 \%)$ & 1.64 & \multirow{3}{*}{$0.019^{*}$} \\
\hline & $13(n=31)$ & $6(19 \%)$ & $18(58 \%)$ & $7(23 \%)$ & 2.03 & \\
\hline & $\mathrm{T} 4(\mathrm{n}=12)$ & $0(0 \%)$ & $6(50 \%)$ & $6(50 \%)$ & 2.50 & \\
\hline \multirow{4}{*}{ Lymph node metastases } & No $(n=16)$ & $7(44 \%)$ & $8(50 \%)$ & $1(6 \%)$ & 1.63 & \multirow{4}{*}{$0.043^{*}$} \\
\hline & $N 1(n=9)$ & $2(22 \%)$ & $5(56 \%)$ & $2(22 \%)$ & 2.00 & \\
\hline & N2 $(n=16)$ & $3(19 \%)$ & $8(50 \%)$ & $5(31 \%)$ & 2.13 & \\
\hline & N3 $(n=13)$ & $0(0 \%)$ & $6(46 \%)$ & $7(54 \%)$ & 2.54 & \\
\hline \multirow{2}{*}{ Distant metastases } & $\mathrm{MO}(\mathrm{n}=42)$ & $12(29 \%)$ & $20(48 \%)$ & $10(24 \%)$ & 1.95 & \multirow{2}{*}{0.070} \\
\hline & M1 $(\mathrm{n}=12)$ & $0(0 \%)$ & $7(58 \%)$ & $5(42 \%)$ & 2.42 & \\
\hline \multirow{2}{*}{ Survival of patients } & Alive $(n=35)$ & $12(34 \%)$ & $20(57 \%)$ & $3(9 \%)$ & 1.74 & \multirow{2}{*}{$<0.001 *$} \\
\hline & Died of cancer $(n=19)$ & $0(0 \%)$ & $7(37 \%)$ & $12(63 \%)$ & 2.63 & \\
\hline
\end{tabular}

* statistically significant when $\mathrm{p}<0.05$

Table 2. The expression of TIMP-1 in interstitial inflammatory infiltrate cells in relation to clinico-pathological features of tumor.

\begin{tabular}{|c|c|c|c|c|c|c|}
\hline \multirow{2}{*}{\multicolumn{2}{|c|}{ Group tested }} & \multicolumn{3}{|c|}{$\operatorname{Cases}(\%)$} & \multirow{3}{*}{$\begin{array}{c}\text { Mcan value } \\
2.14\end{array}$} & \multirow{2}{*}{$\mathrm{p}$} \\
\hline & & \multirow{2}{*}{$\frac{+}{4(29 \%)}$} & \multirow{2}{*}{$\frac{++}{4(29 \%)}$} & \multirow{2}{*}{$\frac{++}{6(43 \%)}$} & & \\
\hline \multirow{3}{*}{$\begin{array}{l}\text { Tumor stage } \\
\text { (INM classification) }\end{array}$} & $\mathrm{I}+\mathrm{Il}(\mathrm{n}=14)$ & & & & & \multirow{3}{*}{$0.001 *$} \\
\hline & III $(n=22)$ & $0(0 \%)$ & $13(59 \%)$ & $9(41 \%)$ & 2.41 & \\
\hline & IV $(n=18)$ & $0(0 \%)$ & $3(17 \%)$ & $15(83 \%)$ & 2.83 & \\
\hline \multirow{3}{*}{ Depth of tumor invasion } & $\mathrm{T} 2(\mathrm{n}=11)$ & $3(27 \%)$ & $3(27 \%)$ & $5(45 \%)$ & 2.18 & \multirow{3}{*}{0.094} \\
\hline & $\mathrm{T} 3(\mathrm{n}=31)$ & $1(3 \%)$ & $14(45 \%)$ & $16(52 \%)$ & 2.48 & \\
\hline & $\mathrm{T} 4(\mathrm{n}=12)$ & $0(0 \%)$ & $3(25 \%)$ & $9(75 \%)$ & 2.75 & \\
\hline \multirow{4}{*}{ Iymph node metastases } & No $(n=16)$ & $4(25 \%)$ & $4(25 \%)$ & $8(50 \%)$ & 2.25 & \multirow{4}{*}{$0.002 *$} \\
\hline & N1 $(n=9)$ & $0(0 \%)$ & $6(67 \%)$ & $3(33 \%)$ & 2.33 & \\
\hline & N2 $(n=16)$ & $0(0 \%)$ & $9(56 \%)$ & $7(44 \%)$ & 2.44 & \\
\hline & N3 $(n=13)$ & $0(0 \%)$ & $1(8 \%)$ & $12(92 \%)$ & 2.92 & \\
\hline \multirow{2}{*}{ Distant metastases } & $\mathrm{MO}(\mathrm{n}=42)$ & $4(10 \%)$ & $19(45 \%)$ & $19(45 \%)$ & 2.36 & \multirow{2}{*}{$0.014 *$} \\
\hline & $\mathrm{M} 1(\mathrm{n}=12)$ & $0(0 \%)$ & $1(8 \%)$ & $11(92 \%)$ & 2.92 & \\
\hline \multirow{2}{*}{ Survival of patients } & $\Lambda$ live $(n=35)$ & $4(11 \%)$ & $19(54 \%)$ & $12(34 \%)$ & 2.23 & \multirow{2}{*}{$<0.001^{*}$} \\
\hline & Died of cancer $(n=19)$ & $0(0 \%)$ & l $(5 \%)$ & $18(95 \%)$ & 2.95 & \\
\hline
\end{tabular}

* statistically significant when $\mathrm{p}<0.05$

tumor (2.0 in stage III and 2.44 in stage IV, respectively) than in stage II (1.64). Similar results were observed in relation to $\mathrm{T}, \mathrm{N}$ and $\mathrm{M}$ factors as well as for survival of patients. The differences in TIMP-1 expression intensity were statistically significant for tumor stage, depth of tumor invasion, nodal involvement and patients' survival.

\section{The expression of tissue inhibitor of matrix metalloproteinases-1 in inflammatory infiltrate cells}

The expression of TIMP-1 in interstitial inflammatory infiltrate cells (macrophages, polinuclears and lymphocytes) in gastric cancer samples was evaluated using the same scale (Fig. 2). A positive reaction was 
Table 3. Results of Cox's univariate analysis in gastric cancer patients.

\begin{tabular}{|l|c|c|}
\hline \multicolumn{1}{|c|}{ Variable analyzed } & OR & $\mathrm{p}$ \\
\hline Giender: M versus I' & 3.023 & 0.139 \\
\hline Age & 1.011 & 0.575 \\
\hline Tumor stage & & $0.009^{*}$ \\
\hline Stage III versus stage II & 1.253 & 0.769 \\
\hline Stage IV versus stage II & 4.933 & $0.015^{*}$ \\
\hline T factor & & 0.133 \\
\hline T3 versus T2 & 0.649 & 0.491 \\
\hline I4 versus I2 & 1.833 & 0.324 \\
\hline N factor & & $0.037^{*}$ \\
\hline N1 versus N0 & 0.977 & 0.984 \\
\hline N2 versus N0 & 2.331 & 0.248 \\
\hline N3 versus N0 & 5.362 & $0.011^{*}$ \\
\hline M factor & 4.996 & $0.001^{*}$ \\
\hline Fxpression of TIMP1 in cancer cells & 4.579 & $0.001^{*}$ \\
\hline $\begin{array}{l}\text { Lxpression of liMP1 in inflammatory } \\
\text { infiltrate cells }\end{array}$ & 17.541 & $0.005^{*}$ \\
\hline
\end{tabular}

* statistically significant when $\mathrm{p}<0.05$

also observed in $100 \%$ of all tissue samples: weak (1 pt) in $7 \%$, moderate ( 2 pts) in $37 \%$ and an intense expression (3 pts) in $56 \%$ of cases.

The results of immunostaining for the presence of TIMP-1 in inflammatory cells in relation to clinicopathological features of tumor are presented in Table 2. We have shown that expression of TIMP-1 in interstitial inflammatory infiltrate cells related to tumor stage. The percentages of intense expression ( 3 pts) of TIMP-1 were the higest in stage IV (83\%) in comparison with less advanced stages ( $43 \%$ in stage II and $41 \%$ in stage III).

The differences in TIMP-1 expression in inflammatory infiltrate cells in relation to depth of tumor invasion ( $\mathrm{T}$ factor) and presence of nodal metastases (N factor) are similar to results observed in cancer cells. The number of intense expression ( $3 \mathrm{pts})$ increased with depth of tumor invasion and nodal involvement. The intense expression was observed more frequently in T4 subgroup (75\%) than in less advanced stages $(45 \%$ in T2 and $52 \%$ in T3) and in N3 samples (92\%) in comparison with subgroup of N0 tumors $(50 \%)$. Immunostaining revealed also the relationships between TIMP-1 expression and presence of distant metastases ( $\mathrm{M}$ factor). There were over two times more cases with intense reactions $(92 \%)$ in the M1 subgroup of GC samples than in M0 tumors (45\%). Moreover, we have observed no cases ( $0 \%$ ) with weak intensity of immunostaining in M1 subgroup in comparison with $10 \%$ in M0 samples.
The relationships between intensity of TIMP-1 expression in inflammatory infiltrate cells and survival of GC patients are similar to those in cancer cells. Intense (3 pts) expression in tumor samples from patients who died of cancer during follow-up period was almost three times higher $(95 \%)$ than in samples from patient who survived (34\%).

The mean values of TIMP-1 were higher in inflammatory infiltrate (2.48) than in cancer cells (2.06). The mean expressions of this protein in inflammatory cells were also higher in more advanced stages of tumor (2.41 in stage III and 2.83 in stage IV, respectively) than in stage II (2.14).

Similar correlations were noticed in relation to $\mathrm{T}$, $\mathrm{N}, \mathrm{M}$ factors and survival of patients. The mean TIMP1 expression in inflammatory cells increased with tumor size, number of lymph nodes involved and in presence of distant metastases as well as in patients died of cancer in comparison with those who survived. The differences in intensity of TIMP-1 expression were statistically significant for tumor stage, nodal involvement, presence of distant metastases and patients' survival. Moreover, the mean values of this protein expression were higher in inflammatory infiltrate cells than in all analyzed subgroups of cancer cells.

\section{Correlations between TIMP-1 expresion in cancer and inflammatory infiltrate cells and prognosis of patients' survival}

Univariate Cox's analysis showed that tumor stage in TNM classification $(\mathrm{p}=0.009)$, presence of lymph node metastases $(\mathrm{N})(\mathrm{p}=0.037)$, distant metastases $(\mathrm{M})$ $(\mathrm{p}=0.001)$ and TIMP-1 expression in cancer $(\mathrm{p}=0.001)$ and inflammatory infiltrate cells $(\mathrm{p}=0.005)$ were statistically significant prognostic factors for patients' survival (Table 3). Consequently, these variables were included in the multivariate regression analysis. It was shown in multivariate analysis that only $\mathrm{T}$ factor $(p=0.023)$ and expression of TIMP-1 in inflammatory infiltrate cells $(\mathrm{p}=0.024)$ were the independent statistically significant prognostic factors affecting survival of GC patients.

\section{Discussion}

Gastric cancer is one of the most aggressive malignant tumors of gastrointestinal tract. It is thought that degradation of extracellular matrix ECM might be an essential step of invasion and metastasis of gastric cancer. MMPs are produced by neoplastic cells and their synthesis in cancer may be induced by cytokines and growth factors [12]. The endogenous inhibitors of matrix metalloproteinases inhibit the active forms of 
MMPs and regulate the processes of their activation. This provides a double negative control of tumor development. The imbalance between MMPs, proteolytic enzymes capable of degrading ECM and their inhibitors may facilitate progression of neoplastic cells.

High expression of TIMP-1 protein in tumor tissues from GC patients was previously shown in several studies $[8,13]$. The concentration of this protein in tumor tissue correlated with patients' survival [14] and more aggresive phenotype of gastric cancer [6]. The expression of TIMP-1 in cancer tissue correlated with clinico-pathological factors, such as depth of tumor invasion $(\mathrm{T})$, nodal metastasis $(\mathrm{N})$ and recurrence of tumor [8]. However, little is known about the relationship between TIMP-1 expression in the interstitial inflammatory infiltrate cells in gastric cancer tissue and clinico-pathological features of tumor and patients' survival.

In the current study, the presence of TIMP-1 in cancer and interstitial inflammatory infiltrate cells was proved by immunostaining and the positive reaction in all specimens was observed. We have shown that TIMP-1 expression in cancer cells depended on tumor stage. The intensity and the mean value of this protein expression were higher in more advanced stages of GC and increased with depth of tumor invasion and presence of lymph node and distant metastases. Our findings are in agreement with results obtained by Seo et al [8], who have shown significant correlation between TIMP-1 expression and T and $\mathrm{N}$ stages in gastric carcinoma samples. Probably the higher expression of TIMP-1 in patients with more advanced gastric cancer might be related to antitumoral response of the organism and predict the aggressive behavior of gastric carcinoma [15].

In the present study, the expression of TIMP-1 in inflammatory cells was assessed. Infiltration of inflammatory cells is a prominent and characteristic feature of many malignant tumors. The inflammatory cells can produce metalloproteinases to the peritumoral environment. These cells are also able to synthesize various cytokines enhancing expression of MMPs by both - tumoral and stromal cells [9]. Intensity of TIMP-1 positive inflammatory infiltrate (macrophages, polynuclears and lymphocytes) in the neoplastic interstitium was evaluated in the same score scale as in cancer cells. The positive reaction in inflammatory infiltrate cells was observed in $100 \%$ of specimens. There was also a tendency to increase the intensity of TIMP-1 expression in advanced stages of tumor in comparison to early stages.

The mean expression of TIMP-1 was higher in inflammatory infiltrate than in cancer cells and increased in more advanced stages of disease. This more intense reaction in advanced stage of disease may be the result of TIMP 1 synthesis by inflammatory cells, i.e. tumor associated macrophages, polynuclear granulocytes and lymphocytes as well as by cancer cells. In cancer tissue, infiltration of inflammatory cells has been suggested to be a mechanism of host resistance [16]. Moreover, we observed that intensity of TIMP-1 expression in both types of cells correlated with patients' survival and TIMP-1 expression in inflammatory cells was an independent, significant prognostic factor for GC patients.

In conclusion, this is the first study comparing the expression of tissue inhibitor of metalloproteinases-1 in inflamatory infiltrate cells in gastric cancer tissue with patients' survival. We proved the presence of TIMP-1 in neoplastic and interstitial inflammatory infiltrate cells in GC tissue and its increased intensity in relation to tumor stage, lymph node metastases and survival of GC patients. The higher incidence of this protein expression in more advanced stages of GC and its correlation with patients' survival suggest the clinical usefulness of TIMP-1 expression as prognostic factor for gastric cancer patients' survival, although this issue requires additional studies.

\section{References}

[1] Alexander HR, Kelsen DP, Tepper JE. Cancer of the stomach. In: DeVita VT, Hellman S, Rosenberg SA (Eds). Cancer. Principles and practice of oncology. $5^{\text {th }}$ Edition. Philadelphia: Lippincott-Raven Publisher, 1997, pp. 1021-22.

[ 2] Seiki M, Yana I. Role of pericellular proteolysis by membrane type-1 matrix metalloproteinase in cancer invasion and angiogenesis. Cancer Sci. 2003;94:569-574.

[3] Crawford HC, Matrisian LM. Tumor and stromal expression of matrix metalloproteinases and their role in tumor progression. Invasion Metastasis. 1995;14:234-245.

[4] Wagenaar-Miller RA, Gorden L, Matrisian LM. Matrix metalloproteinases in colorectal cancer: is it worth talking about? Cancer Metastasis Rev. 2004;23:119-135.

[ 5] Gomez DE, Alonso DF, Yoshiji H, Thorgeirsson UP. Tissue inhibitors of metalloproteinases: structure, regulation and biological functions. Eur J Cell Biol. 1997;74:111-122.

[ 6] De Mingo M, Morán A, Sánchez-Pernaute A et al. Expression of MMP-9 and TIMP-1 as prognostic markers in gastric carcinoma. Hepatogastroenterol. 2007;54:315-319.

[ 7] Işlekel H, Oktay G, Terzi C, Canda AE, Füzün M, Küpelioglu A. Matrix metalloproteinase-9, -3 and tissue inhibitor of matrix metalloproteinase-1 in colorectal cancer: relationship to clinicopathological variables. Cell Biochem Funct. 2007; 25:433-441.

[ 8] Seo YS, Park JJ, Kim JH et al. Usefulness of MMP-9/TIMP1 in predicting tumor recurrence in patients undergoing curative surgical resection for gastric carcinoma. Dig Dis Sci. 2007;52:753-759.

[ 9] Biswas C, Zhang Y, DeCastro R et al. The human tumor cellderived collagenase stimulatory factor (renamed EMMPRIN) is a member of the immunoglobulin superfamily. Cancer Res. 1995;55:434-439.

[10] Jass JR, Sobin LH. WHO International Histological Classification of Tumors. Histological typing of Intestinal Tumors. New York, Springer-Verlag, 1989. 
[11] Mehta CR, Patel NR. Algorithm 643: FEXACT: A FORTRAN subroutine for Fisher's exact test on unordered $\mathrm{r} \times$ c contingency tables. ACM Transactions on Mathematical Software.1986;12:154-161.

[12] Bonomi P. Matrix metalloproteinases and matrix metalloproteinase inhibitors in lung cancer. Semin Oncol. 2002;29:78-86.

[13] Zhang S, Li L, Lin JY, Lin H. Imbalance between expression of matrix metalloproteinase- 9 and tissue inhibitor of metalloproteinase-1 in invasiveness and metastasis of human gastric carcinoma. World J Gastroenterol. 2003;9:899-904.

[14] Yoshikawa T, Tsuburaya A, Kobayashi O, Sairenji M, Miyagi Y. Protein levels of tissue inhibitor of metalloproteinase-1 in tumor extracts as a marker for prognosis and recurrence in patients with gastric cancer. Gastric Cancer. 2006;9:106-113.

[15] Joo YE, Seo KS, Kim HS, Rew JS, Park CS, Kim SJ. Expression of tissue inhibitors of metalloproteinases (TIMPs) in gastric cancer. Dig Dis Sci. 2000;45:114-121.

[16] Suzuki Y, Ohtani H, Mizoi T et al. Cell adhesion molecule expression by vascular endothelial cells as an immune/inflammatory reaction in human colon carcinoma. Jpn J Cancer Res. 1995;86:585-593.

Submitted: 22 April, 2009 Accepted after reviews: 27 July, 2009 